\title{
Projection Volumes of Hyperplane Arrangements
}

\author{
Caroline J. Klivans • Ed Swartz
}

Received: 11 February 2010 / Revised: 25 April 2011 / Accepted: 10 May 2011 /

Published online: 1 July 2011

(C) Springer Science+Business Media, LLC 2011

\begin{abstract}
We prove that for any finite real hyperplane arrangement the average projection volumes of the maximal cones are given by the coefficients of the characteristic polynomial of the arrangement. This settles the conjecture of Drton and Klivans that this held for all finite real reflection arrangements. The methods used are geometric and combinatorial. As a consequence, we determine that the angle sums of a zonotope are given by the characteristic polynomial of the order dual of the intersection lattice of the arrangement.
\end{abstract}

Keywords Angle sum - Characteristic polynomial - Hyperplane arrangement · Zonotope

\section{Introduction}

Given a polyhedral cone $\mathcal{C} \subseteq \mathbb{R}^{n}$, consider the orthogonal projection of an arbitrary point $\mathbf{z} \in \mathbb{R}^{n}$ onto $\mathcal{C}$. The work here is concerned with the dimension of the face $\mathbf{z}$ projects onto. Specifically, consider the following problem as formulated in [4]:

Problem 1 Which fraction of the unit sphere in $\mathbb{R}^{n}$, as measured by surface volume, is occupied by the points $\mathbf{z}$ for which the orthogonal projection of $\mathbf{z}$ onto $\mathcal{C}$ lies in the interior of a $k$-dimensional face of $\mathcal{C}$ ?

The work here was done while the first author was a visiting scholar in the mathematics department at Cornell University. The second author is partially supported by NSF grant DMS-0900912.

C.J. Klivans ( $\square)$

Departments of Mathematics and Computer Science, The University of Chicago, Chicago, USA

e-mail: cjk@math.uchicago.edu

E. Swartz

Department of Mathematics, Cornell University, Ithaca, USA 
The study of these projection volumes is motivated by $p$-value calculations in statistical hypothesis testing; see [4] for more on this motivation. There the projection volumes of fundamental chambers of finite real reflection arrangements are investigated. It is shown that for irreducible reflection groups of type $A_{n}, B_{n}$ and $D_{n}$, the projection volumes are given by the coefficients of the characteristic polynomial of the corresponding reflection arrangement. These results are natural extensions of those shown by De Concini, Procesi, Stembridge, and Denham [2, 3] for zero dimensional projections onto reflection arrangements.

Here we extend and strengthen these results. In particular, we offer a positive answer to the main Conjecture of [4] which states that the projection volumes of any finite real reflection arrangement are given by the coefficients of its characteristic polynomial.

This follows as a corollary of a stronger result, our Theorem 5, which considers projections onto cones formed from regions of arbitrary finite real hyperplane arrangements. In order to understand this more general case, we shift our perspective and consider the average projection volumes over all regions of a given hyperplane arrangement. Theorem 5 states that for an arbitrary finite real hyperplane arrangement the average projection volumes are given by the coefficients of its characteristic polynomial. As all regions of a reflection arrangement are isometric, Corollary 5 of [4] holds for all finite real reflection groups.

The methods utilized in [4] are algebraic, drawing on the structure of a reflection group. In contrast, here we use combinatorial and geometric techniques to prove our main result. Projection volumes of cones are related to the characteristic polynomial through the theory of polytope angle sums. To any linear hyperplane arrangement there are naturally associated dual polytopes, called zonotopes. A well-known result of Zaslavsky gives the face numbers of zonotopes in terms of the Möbius function of the intersection lattice of the arrangement. A little known result of Perles and Shephard then relates the face numbers of a zonotope to its angle sums. Combining these results, Theorem 5 equates the angle sums of certain faces of the zonotopes to both the value of the Möbius function at a given intersection and the projection volume onto that intersection. We further determine that the angle sums of a zonotope are given by the characteristic polynomial of the order dual of the lattice of flats of the arrangement.

\section{Projection Volumes}

Let $\mathcal{C}$ be a polyhedral set in $\mathbb{R}^{d}$. Unless otherwise noted, $\mathcal{C}$ will always be a cone. For an arbitrary point $\mathbf{z} \in \mathbb{R}^{d}$, let $\pi_{\mathcal{C}}(\mathbf{z})$ be the orthogonal projection of $\mathbf{z}$ onto $\mathcal{C}$, i.e., $\pi_{\mathcal{C}}$ is the nearest point map. Say that $\pi_{\mathcal{C}}(\mathbf{z})$ has $k$-dimensional projection if $\pi_{\mathcal{C}}(\mathbf{z})$ lies in the relative interior of a $k$-dimensional face of $\mathcal{C}$. Define $v_{k}$ to be the ratio of volume of $\mathbb{R}^{d}$ occupied by points $\mathbf{x}$ for which the projection $\pi_{\mathcal{C}}$ is $k$-dimensional. There are several ways of making this precise. Let $X$ be a cone. Define the volume of $X$ using any of the following equivalent definitions: 
Volume of $X=$ The ratio of volume of $\mathbb{R}^{d}$ occupied by $X$

$$
\begin{aligned}
& =\frac{\left|X \cap S^{d-1}\right|}{\left|S^{d-1}\right|} \\
& =\frac{\left|X \cap B^{d}\right|}{\left|B^{d}\right|},
\end{aligned}
$$

where $|\cdot|$ is the Lebesgue measure, $S^{d-1}$ is the unit sphere, and $B^{d}$ is the unit ball.

The set of points $\mathbf{z}$ which project into the relative interior of a face $F$ of $\mathcal{C}$ is a cone which we denote by $X_{F}$. Set $v(F)=$ the volume of $X_{F}$. If $F$ is contained in several polyhedral sets, then we use $v_{\mathcal{C}}(F)$ to specify which one. Finally, we define $v_{k}$ to be the sum of the $v(F)$ over all $k$-dimensional faces $F$ of $\mathcal{C}$. The $v_{k}$ are called the projection volumes of $\mathcal{C}$. The work here is motivated by trying to determine the $v_{k}$ for a given cone.

Example 2 [4, Example 2] Consider the cone $\mathcal{C}=[0, \infty)^{2}$ equal to the non-negative orthant in $\mathbb{R}^{2}$. All points $\mathbf{z}$ in the positive orthant $(0, \infty)^{2}$ lie inside the cone and thus have a 2-dimensional projection $\pi_{\mathcal{C}}(z)$. All points in the non-positive orthant $(-\infty, 0]^{2}$, the polar cone, are projected to the origin, that is, they have 0 -dimensional projection. As all remaining points have 1-dimensional projection, the projection volumes are $v_{0}=v_{2}=1 / 4$ and $v_{1}=1 / 2$.

We will consider polyhedral sets formed by hyperplane arrangements. We review the basics of the combinatorics of hyperplane arrangements and refer the reader to [11] for much more. A real hyperplane arrangement $\mathcal{A}$ is a collection of codimension-one affine subspaces of $\mathbb{R}^{d}$. All arrangements appearing in this paper are assumed finite. The rank of an arrangement $\mathcal{A}$ is defined to be the dimension of the linear space spanned by the normal vectors to its hyperplanes. Specifically, if $\mathcal{A}=\left\{H_{1}, \ldots, H_{m}\right\}$ and $H_{i}=\left\{\mathbf{z} \in \mathbb{R}^{d}: \eta_{i} \cdot \mathbf{z}=b_{i}\right\}$, where $\eta_{i}$ is a non-zero vector in $\mathbb{R}^{d}$, then

$$
\operatorname{Rk}(\mathcal{A})=\operatorname{dim}\left(\operatorname{Span}\left\{\eta_{1}, \ldots, \eta_{m}\right\}\right) .
$$

A region of $\mathcal{A}$ is any connected component of the complement of the union of all the hyperplanes in $\mathcal{A}$. We denote by $\mathcal{R}(\mathcal{A})$ the set of all regions of $\mathcal{A}$. When all hyperplanes pass through the origin, and hence the closure of any region forms a polyhedral cone, the arrangement is called central. Generally, the closure of any region forms a polyhedra. Note that any polyhedral cone can be thought of as a region in the hyperplane arrangement formed by taking the bounding hyperplanes of the cone.

Much of the combinatorics of a hyperplane arrangement is encoded by its intersection poset. Given an arrangement $\mathcal{A}$, let $L(\mathcal{A})$ be the set of all nonempty intersections of collections of hyperplanes in $\mathcal{A}$. We include $\mathbb{R}^{d}$ in $L(\mathcal{A})$ as the intersection of the empty collection. Define a partial order on $L(\mathcal{A})$ by reverse inclusion of intersections, that is, $x \leq y$ in $L(A)$ if $y \subseteq x$. Then $L(\mathcal{A})$ forms a poset ranked by codimension $d-\operatorname{dim}(x)$. If the arrangement is central, then $L(\mathcal{A})$ contains a unique top element $\hat{1}$ and forms a lattice. If it is also the case that $\hat{1}=\{0\}$, then the arrangement is called essential. 
The Möbius function $\mu$ of a finite poset $P$ is a function from intervals of $P$ to $\mathbb{Z}$ defined recursively by:

$$
\begin{aligned}
& \mu(x, x)=1, \quad \text { for all } x \in P, \\
& \mu(x, y)=-\sum_{x \leq z<y} \mu(x, z), \quad \text { for all } x<y \in P .
\end{aligned}
$$

Write $\mu(x)$ for $\mu(\hat{0}, x)$ when $P$ has a minimal element $\hat{0}$. The characteristic polynomial of a rank $r$ graded poset with $\hat{0}$ and rank function $\rho$ is defined as

$$
\chi_{P}(t)=\sum_{x \in P} \mu(x) t^{r-\rho(x)} .
$$

If $\mathcal{A}$ is an essential central arrangement, then for $L(\mathcal{A})$ this equals

$$
\chi_{\mathcal{A}}(t)=\sum_{x \in L(\mathcal{A})} \mu(x) t^{\operatorname{dim} x} .
$$

Example 3 Consider the hyperplane arrangement $\mathcal{A} \subset \mathbb{R}^{2}$ consisting of any three lines $H_{1}, H_{2}$, and $H_{3}$ through the origin. The intersection lattice of this arrangement is $L(\mathcal{A})=\left\{\mathbb{R}^{2}, H_{1}, H_{2}, H_{3},\{0\}\right\}$ with its elements ordered as $\mathbb{R}^{2} \leq H_{1} \leq\{0\}, \mathbb{R}^{2} \leq$ $H_{2} \leq\{0\}$, and $\mathbb{R}^{2} \leq H_{3} \leq\{0\}$. The Möbius function thus assigns the values $\mu\left(\mathbb{R}^{2}\right)=$ $1, \mu\left(H_{1}\right)=\mu\left(H_{2}\right)=\mu\left(H_{3}\right)=-1$ and $\mu(\{0\})=2$. The characteristic polynomial equals $\chi_{\mathcal{A}}(t)=t^{2}-3 t+2$.

In order to understand projection volumes, we will shift our perspective and consider the average projection volumes over all regions of a given hyperplane arrangement.

Example 4 For any three lines passing through the origin in $\mathbb{R}^{2}$, the average twodimensional volume will always be $\frac{1}{6}$ as there are 6 regions. To determine the zerodimensional volume of a cone, consider the interior angle $\alpha$ of the cone. The fraction of volume which projects onto the vertex of the cone is $\frac{1}{2}-\alpha$. Thus the average volumes are $\left(\frac{1}{6}, \frac{1}{2}, \frac{1}{3}\right)$.

Our main result, Theorem 5, relates the sum of projection volumes to the characteristic polynomial. Before stating the theorem, we recall a well-known result due to Zaslavsky [12] and, independently, Las Vergnas [7]:

$$
(-1)^{r} \chi_{\mathcal{A}}(-1)=|\mathcal{R}(\mathcal{A})|,
$$

where $|\mathcal{R}(\mathcal{A})|$ denotes the number of regions of $\mathcal{A}$. In particular, as the coefficients of $\chi_{\mathcal{A}}$ alternate in sign [9, Theorem 4, p. 357], the sum of the absolute values of the coefficients of $\chi_{\mathcal{A}}(t)$ equals the number of regions of $\mathcal{A}$.

Theorem 5 Let $\mathcal{A}$ be a rank $r$ real hyperplane arrangement in $\mathbb{R}^{d}$. Then the sum $\sum_{\mathcal{C}} v_{k}(\mathcal{C})$ over all regions $\mathcal{C}$ of $\mathcal{A}$ is equal to the absolute value of the coefficient of $t^{r-d+k}$ of the characteristic polynomial of $\mathcal{A}$. 


\section{Zonotopes}

The link between projection volumes of regions of hyperplane arrangements and coefficients of characteristic polynomials is via zonotopes. Zonotopes are a rich class of polytopes equivalently defined as affine projections of cubes, Minkowski sums of line segments, or polytopes with all faces centrally symmetric.

Zonotopes naturally arise from any central hyperplane arrangement. Throughout this section, $\mathcal{A}$ will always be an essential central arrangement. Consider the face lattice $F(\mathcal{A})$ of such an arrangement $\mathcal{A}$. This poset records the cellular structure of the decomposition of space as induced by the hyperplanes. Since the arrangement is essential and central, $F(\mathcal{A})$ has a unique bottom element corresponding to the origin, the atoms correspond to one-dimensional rays and in general the $j$-dimensional cones are represented by rank $j$ elements of $F(\mathcal{A})$. Next consider a zonotope $Z$ formed by taking the Minkowski sum of normals of all the hyperplanes in $\mathcal{A}$. Different choices of normals lead to geometrically distinct zonotopes, but they are all combinatorially equivalent. Indeed, let $F(Z)$ be the face lattice of $Z$. Then $F(Z)$ is isomorphic to the order dual of $F(\mathcal{A})$ union 0 ; see, for instance, [13, Chapter 7]. Informally, for each region of $\mathcal{A}$ we have a vertex of $Z$, for each pair of neighboring regions we have an edge of $Z$, etc.

We will relate projection volumes of regions of $\mathcal{A}$ to angles associated to the zonotope $Z$. As an example, consider $v_{0}(\mathcal{C})$ the zero dimensional projection onto a region $\mathcal{C}$ of $\mathcal{A}$. The set of points $\mathbf{x}$ such that $\pi_{\mathcal{C}}(\mathbf{x})=0$ is given by the normal cone of $\mathcal{C}$, i.e., the cone generated by all opposites of normals of hyperplanes supporting $\mathcal{C}$. The normal cone is a translate of the cone induced by the corresponding vertex figure of $Z$, see Fig. 1 .

In general, the normal fan of $Z$ formed by taking the normal cones to all faces of $Z$ is equal to the face fan of $\mathcal{A}$. As expected by the duality described above, we have even more generally that the fan of the arrangement is equal to the face fan of the polar of the zonotope, see [13, Corollary 7.18].

Fig. 1 An arrangement of three lines with the corresponding zonotope. The normal cone to $\mathcal{C}$ is given by the dotted rays emanating at the origin. The cone prescribed by the vertex figure sitting inside $\mathcal{C}$ is a translate of the normal cone

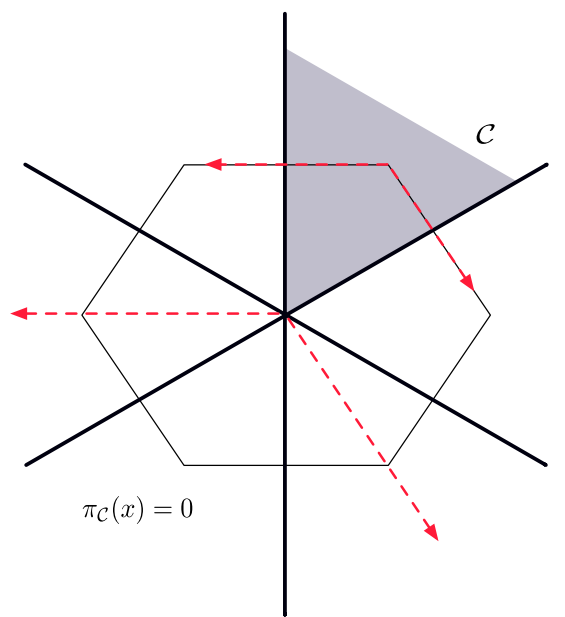




\section{Angle Sums of Polytopes}

In order to use zonotopes to understand $k$-dimensional projections, we will need the theory of angle sums of polytopes. Let $P$ be a polytope, $F$ a face of $P$ and $z$ a point in the relative interior of $F$. Define the angle of $P$ at $F, \alpha(P, F)$, as the ratio of volume of an epsilon-ball centered at $\mathbf{z}$ which lies inside $P$. Specifically, if $B$ is a sufficiently small ball centered at $\mathbf{z}$, then $\alpha(P, F)$ is the ratio of volume of $B \cap P$ to the volume of $B$.

Example 6 We have $\alpha(P, F)=1$ for the improper face $F=P$ of $P$ and $\alpha(P, G)=\frac{1}{2}$ for every facet $G$ of $P$.

Define the $k$ th angle sum of $P, \alpha_{k}(P)$, as the sum of all angles over faces of dimension $k$ :

$$
\alpha_{k}(P)=\sum_{\operatorname{dim} F=k} \alpha(P, F)
$$

Example 7 For every $d$-dimensional polytope $P$, we have $\alpha_{d}(P)=1$ and $\alpha_{d-1}(P)=$ $\frac{1}{2} f_{d-1}$, where $f_{d-1}$ denotes the number of $(d-1)$-dimensional faces of $P$.

Angle sums of polytopes satisfy relations similar to those satisfied by the face numbers of a polytope, where the face numbers $f(P)=\left(f_{0}, f_{1}, \ldots, f_{d-1}\right)$, also called the $f$-vector, record the number of faces in each dimension; see, for example, [1] and [8]. Furthermore, there are strong connections between the angle sums and the $f$-vector of a given polytope. We review here a result of Perles and Shephard relating angle sums and face numbers for the class of equiprojective polytopes.

Equiprojective polytopes are polytopes such that all projections onto sufficiently generic hyperplanes have the same $f$-vector. Formally, let $P_{\mathbf{z}}$ be the polytope obtained by orthogonally projecting $P$ onto the hyperplane with normal $\mathbf{z}$. A polytope is called equiprojective if the face numbers of $P_{\mathbf{z}}$ are the same for all $\mathbf{z}$ not parallel to any face of $P$.

Theorem 8 (Perles-Shephard [8]) Let $P$ be a d-dimensional equiprojective polytope and $P^{\prime}$ a generic projection of $P$. Then for $0 \leq k \leq d-1$

$$
\alpha_{k}(P)=\frac{1}{2}\left(f_{k}(P)-f_{k}\left(P^{\prime}\right)\right)
$$

where we set $f_{d-1}\left(P^{\prime}\right)=0$.

This theorem is actually a corollary of a more general result in the same paper. Let $\alpha(P)=\left(\alpha_{0}, \ldots, \alpha_{d-1}\right)$. Perles and Shephard prove that for any polytope the vector $f(P)-2 \alpha(P)$ is a convex combination of all of the possible $f$-vectors of the projections $P_{\mathbf{z}}$. The proof of this theorem relies on two simple, yet powerful ideas. The first is that $\alpha(P, F)$ is one-half of the measure of the normals $\mathbf{z}$ such that the projected image of $F$ is not a face of $P_{\mathbf{z}}$. The other is that we can compute the expected number of faces in a projection $P_{\mathbf{z}}$ by either integrating over the unit sphere 
the number of faces for each $P_{\mathbf{z}}$, or by using the above interpretation of $\alpha(P, F)$. Anyone unfamiliar with these ideas is strongly encouraged to begin by reading the elementary, yet revealing [5]

Theorem 9 (Shephard [10]) Zonotopes are equiprojective.

In order to use the above theorems, we need to understand $f_{k}(P)$ and $f_{k}\left(P^{\prime}\right)$ for a zonotope $P$. If $P$ is $d$-dimensional, $f_{0}(P)$ can be computed from the corresponding arrangement by (1). The general formula is as follows.

Theorem 10 (Zaslavsky [12]) Let $\mathcal{A}$ be an arrangement of hyperplanes in $\mathbb{R}^{d}$. Then the face numbers of any zonotope of $\mathcal{A}$ are given by

$$
f_{k}=\sum_{\operatorname{dim} x=d-k} \sum_{x \leq y}(-1)^{\operatorname{dim} x-\operatorname{dim} y} \mu(x, y) .
$$

In particular, setting $k=0$ recovers (1).

Lemma 11 Let $\mathcal{A}$ be an essential central hyperplane arrangement in $\mathbb{R}^{d}$ and let $Z$ be an associated zonotope. Then $\alpha_{0}(Z)=\left|\mu_{L(\mathcal{A})}(\hat{0}, \hat{1})\right|$.

Proof Write the characteristic polynomial of $\mathcal{A}$ as

$$
\chi_{\mathcal{A}}(t)=a_{0} t^{d}-a_{1} t^{d-1}+\cdots+(-1)^{d-2} a_{d-2} t^{2}+(-1)^{d-1} a_{d-1} t+(-1)^{d} a_{d},
$$

where all the $a_{i}$ are nonnegative. (In fact, they are all positive.) As noted above, the vertices of $Z$ correspond to the regions of $\mathcal{A}$, so (1) tells us that

$$
f_{0}(Z)=a_{0}+a_{1}+\cdots+a_{d-2}+a_{d-1}+a_{d} .
$$

Let $Z^{\prime}$ be the projection of $Z$ into a generic hyperplane $x$. What is $f_{0}\left(Z^{\prime}\right)$ ? Evidently, $Z^{\prime}$ is the Minkowski sum of the images of the projections of the normals $\eta_{i}$ which determined $Z$, and hence is a $(d-1)$-dimensional zonotope. Denote the images of the normals by $\eta_{i}^{\prime}$ and the associated arrangement by $\mathcal{A}^{\prime}$. The intersection poset of any arrangement of linear hyperplanes is completely determined by the dimensions of the various intersections of the hyperplanes or, equivalently, by the ranks of all the possible subsets of normal vectors. The choice of $x$ ensures that for any subset of the $\eta_{i}$ its rank is the same as the corresponding subset of $\eta_{i}^{\prime}$ except when the former has rank $d$. In that case the rank of the $\eta_{i}^{\prime}$ is only $d-1$. From this we see that $L\left(\mathcal{A}^{\prime}\right)$ is just the truncation of $L(\mathcal{A})$. Specifically, $L\left(\mathcal{A}^{\prime}\right)$ is $L(\mathcal{A})$ with its coatoms removed.

Now write the characteristic polynomial of $\mathcal{A}^{\prime}$ as $\chi_{\mathcal{A}^{\prime}}(t)=b_{0} t^{d-1}-b_{1} t^{d-2}+\cdots+(-1)^{d-3} b_{d-3} t^{2}+(-1)^{d-2} b_{d-2} t+(-1)^{d-1} b_{d-1}$, where all of the $b_{i}$ are positive. Since $L(\mathcal{A})$ and $L\left(\mathcal{A}^{\prime}\right)$ agree up to rank $d-2, a_{i}=b_{i}$ for all $0 \leq i \leq d-2$. Theorems 8, 9 and 10 imply that $\alpha_{0}(Z)=\frac{1}{2}\left(a_{d-1}+a_{d}-b_{d-1}\right)$. Since the alternating sums of the $a_{i}$ and the $b_{j}$ are zero, as the Möbius values of a lattice with $\hat{1}, a_{d-1}-a_{d}=b_{d-1}$. By definition, $a_{d}=\left|\mu_{L(\mathcal{A})}(\hat{0}, \hat{1})\right|$. 
Theorem 12 Let $\mathcal{A}$ be an essential central hyperplane arrangement and let $Z$ be an associated zonotope. Then $\alpha_{i}(Z)$ is the coefficient of $t^{i}$ in the characteristic polynomial of the order dual of $L(\mathcal{A})$.

Proof Applying the same reasoning as in the proof of the above lemma, we find that

$$
\alpha_{i}(Z)=\sum_{\operatorname{dim} x=d-i} \mu_{L(\mathcal{A})}(x, \hat{1}) .
$$

The result follows as $\mu_{L(\mathcal{A})}(x, \hat{1})=\mu_{L_{\mathcal{A}}^{*}}(\hat{0}, x)$.

As pointed out by one of the anonymous referees, Lemma 11 and the above theorem are closely related to [6, Theorem 3.1 and Theorem 3.5], results of Greene and Zaslavsky. In fact, a proof of the main theorem can be presented as a combination of the Greene-Zaslavsky results and the ideas of the Perles-Shephard paper.

\section{Proof of the Main Theorem}

We are now ready to prove our main theorem.

Theorem 5. Let $\mathcal{A}$ be a central rank $r$ real hyperplane arrangement in $\mathbb{R}^{d}$. Then the sum $\sum_{\mathcal{C}} v_{k}(\mathcal{C})$ over all regions $\mathcal{C}$ of $\mathcal{A}$ is given by the absolute value of the coefficient of $t^{r-d+k}$ of the characteristic polynomial of $\mathcal{A}$.

Proof Our first observation is that it is sufficient to prove this for essential arrangements. Indeed, suppose that $\operatorname{dim} \hat{1}=d-r>0$. Let $V$ be the orthogonal complement of $\hat{1}$ and consider the hyperplane arrangement $\mathcal{A}_{V}=\left\{H_{1} \cap V, \ldots, H_{m} \cap V\right\} \subseteq V$. Now, $L\left(\mathcal{A}_{V}\right) \cong L(\mathcal{A})$, so their characteristic polynomials are identical. Furthermore, the regions of $\mathcal{A}_{V}$ correspond bijectively to the regions of $\mathcal{A}$ by $C \cap V \leftrightarrow C$ and $v_{k}(C \cap V)=v_{k+d-r}(C)$. Thus the theorem holds for $\mathcal{A}$ by applying it to the essential arrangement $\mathcal{A}_{V}$. Hence, from here on we will assume that $\mathcal{A}$ is an essential central arrangement.

Let $x \in L(\mathcal{A})$ and let $\mathcal{F}_{x}$ be the set of faces of $\mathcal{A}$ whose affine span is $x$. Our second observation is that it is sufficient to prove

$$
\sum_{F \in \mathcal{F}_{x}} \sum_{\substack{F \subseteq \mathcal{C} \\ \mathcal{C} \in \mathcal{\mathcal { R }}(\mathcal{A})}} v_{\mathcal{C}}(F)=\mu_{L(\mathcal{A})}(x) .
$$

To see that this is sufficient, note that $v_{k}$ is the sum over all pairs $v_{\mathcal{C}}(F)$ with $\operatorname{dim} F=k, \mathcal{C} \in \mathcal{R}(\mathcal{A})$ and $F \subseteq \mathcal{C}$. Each such face $F$ is contained in exactly one $\mathcal{F}_{x}$ with the rank of $x$ equal to $d-k$, and the sum of the $\mu_{L(\mathcal{A})}(x)$ of rank $d-k$ is the required coefficient of the characteristic polynomial.

Fix $Z$ a zonotope for $\mathcal{A}$. Recall that an element of the intersection lattice $x \in L(\mathcal{A})$ corresponds to a collection of parallel faces of $Z$, the duals of the cones $F \in \mathcal{F}_{x}$. Each 
of the faces in the zonotope corresponding to $x$ is formed by taking the sum:

$$
\sum \lambda_{i} \eta_{i}:-1 \leq \lambda_{i} \leq 1
$$

over all hyperplanes $H_{i}=\left\{\mathbf{z} \in \mathbb{R}^{d}: \eta_{i} \mathbf{z}=0\right\}$ in $x$ and translating by some \pm 1 combinations of the $\eta_{i}$ corresponding to hyperplanes not in $x$ [13, Chap. 7]. In particular, all of these faces are isometric.

Let $Z_{x}$ be one of the isometric faces of $Z$ corresponding to the intersection $x$ and let $\mathcal{A}_{x}$ be the subarrangement of $\mathcal{A}$ consisting of those hyperplanes which contain $x$. For any $x<\hat{1}, \mathcal{A}_{x}$ is a non-essential arrangement in $\mathbb{R}^{d}$ but forms an essential central arrangement if we project onto $V_{x}$, the orthogonal complement of $x$. The lattice of the projected arrangement is isomorphic to the interval $[\hat{0}, x]$ in the original lattice. Furthermore, we see by the form of $Z_{x}$ given above, a zonotope corresponding to the projected arrangement is simply (a translate of) $Z_{x}$. Lemma 11 then equates the vertex angle sum of $Z_{x}$ and the Möbius value at the intersection $x$ :

$$
\alpha_{0}\left(Z_{x}\right)=\mu_{L(\mathcal{A})}(\hat{0}, x) .
$$

This gives the vertex angle sum of $Z_{x}$ in terms of the Möbius function at $x$. Let $F \in F(\mathcal{A})$ be the normal cone to $Z_{x}$. Next we relate the vertex angle sum of $Z_{x}$ with $\sum_{\mathcal{C} \in \mathcal{R}(\mathcal{A})} v_{\mathcal{C}}(F)$

For a particular $\mathcal{C}$, for which points $\mathbf{y} \in \mathbb{R}^{d}$ is $\pi_{\mathcal{C}}(\mathbf{y}) \in F$ ? The set of all such points form a polyhedral cone which we previously denoted $X_{F}$. This projection cone is given by the positive span of the normal cone in $V_{x}$ of the origin with respect to the projection of $C$ to $V_{x}$ and $F$ itself. Since these cones lie in orthogonal subspaces they form a product cone.

Let $w$ be the vertex of (the translated) $Z_{x}$ which corresponds by duality to $\mathcal{C}$. As the normal fan of $Z_{x}$ in $V_{x}$ is $\mathcal{A}_{x}$, its normal cone is isometric to the cone used to determine $\alpha\left(Z_{x}, w\right)$, the angle sum of $Z_{x}$ at $w$. Thus $v_{\mathcal{C}}(F)=\alpha\left(Z_{x}, w\right)$. By duality, for a fixed $F$, there is a one-to-one correspondence between pairs $F \subseteq \mathcal{C}$ and the vertices of $Z_{x}$. Hence, by Lemma 11 , for a particular $F$,

$$
\sum_{F \subseteq \mathcal{C} \in \mathcal{R}(\mathcal{A})} v_{\mathcal{C}}(F)=\mu_{L(\mathcal{A})}(x) .
$$

As the set of all of the interiors $F \in \mathcal{R}\left(\mathcal{A}_{x}\right)$ form an open dense subset of $V_{x}$ of full measure and as previously noted the normal cones are all products, (2) holds and we are done.

Theorem 5 can be shown to hold in the greater generality of all finite affine arrangements. As we know of no applications, we only sketch the proof. The main difficulty in this setting is extending the notion of projection volume. The regions of affine arrangements can be arbitrary polyhedral sets and hence the definition of $v(F)$ from Sect. 2 is no longer adequate. Instead, we again let $X_{F}$ be the set of all points $\mathbf{z} \in \mathbb{R}^{d}$ such that $\pi_{C}(\mathbf{z})$ is in the interior of $F$. But now we define

$$
v_{\mathcal{C}}(F)=\lim _{r \rightarrow \infty} \frac{\left|B_{r}(\mathbf{a}) \cap X_{F}\right|}{\left|B_{r}(\mathbf{a})\right|},
$$


where $B_{r}(\mathbf{a})$ is the ball of radius $r$ around a fixed point $\mathbf{a} \in \mathbb{R}^{d}$. This is necessary, for example, for bounded faces of the arrangement which are not zero-dimensional. Such bounded faces in fact have zero projection volumes. After proving that the above limit exists and is independent of $\mathbf{a}$ the argument in the proof of the main theorem can be repeated.

\section{Isometric Regions}

As an obvious corollary to Theorem 5 we see that for an arrangement with all isometric regions, the coefficients of the characteristic polynomial give the precise projection volumes for any fixed region.

Reflection (or Coxeter) arrangements constitute a large class of such arrangements. In [4], projection volumes of reflection arrangements were studied directly heavily utilizing the structure of the reflection group. The corollary above was established for certain families of reflection groups and conjectured for all reflection groups. Theorem 5 now affirmatively answers this conjecture.

We leave as an open question whether or not there exist other examples of arrangements with isometric cones.

Problem 13 Does there exists a real central hyperplane arrangement with all cones isometric that is not a reflection arrangement?

Acknowledgement We thank the anonymous referees for several suggestions in improving the exposition. Especially, the observations concerning the Greene-Zaslavsky results in [6].

\section{References}

1. Camenga, K.: Vector spaces spanned by the angle sums of polytopes. Beiträge Algebra Geom. 47(2), 447-462 (2006)

2. De Concini, C., Procesi, C.: A Curios Identity and the Volume of the Root spherical simplex. Atti Accad. Naz. Lincei Cl. Sci. Fis. Mat. Natur. Rend. Lincei (9) Mat. Appl. With an appendix by J. Stembridge. (17) (2006)

3. Denham, G.: A Note on De Concini and Procesi's Curios Identity. Atti Accad. Naz. Lincei Cl. Sci. Fis. Mat. Natur. Rend. Lincei (9) Mat. Appl. (19) (2008)

4. Drton, M., Klivans, C.: A geometric interpretation of the characteristic polynomial of reflection arrangements. Proc. Am. Math. Soc. 138(8) (2010)

5. Felman, D., Klain, D.: Angles as probabilities. Am. Math. Monthly 116 (2009)

6. Greene, C., Zaslavsky, T.: On the interpretation of Whitney numbers through arrangements of hyperplanes, zonotopes, non-radon partitions and orientations of graphs. Trans. Am. Math. Soc. 280 (1983)

7. Vergnas, M. Las: Convexity in oriented matroids. J. Combin. Theory, Ser. B 29 (1980)

8. Perles, M.A., Shephard, G.C.: Angle sums of convex polytopes. Math. Scand. 21 (1967)

9. Rota, G.-C.: On the foundations of combinatorial theory I. Theory of Möbius functions. Z. Wahrscheinlichkeitstheor. Verw. 2 (1964)

10. Shephard, G.C.: Polytopes with centrally symmetric faces. Canad. J. Math. 19 (1967)

11. Stanley, R.: An introduction to hyperplane arrangements. In: Geometric Combinatorics. IAS/Park City Math., vol. 13. Amer. Math. Soc., Providence (2007)

12. Zaslavsky, T.: Facing up to arrangements: face-count formulas for partitions of space by hyperplanes. Mem. Amer. Math. Soc. 1(1), 154 (1975)

13. Ziegler, G.: Lectures on Polytopes. Graduate Texts in Mathematics, vol. 152. Springer, New York (1995) 